\title{
Qualité des soins
}

\section{P. Garnerin,$^{b, g}$ P. Bovier, ${ }^{b, d}$ E. Chamot, ${ }^{a}$ P. Chastonay, ${ }^{a, c}$ \\ P. Chopard,,$^{b, f}$ F. Herrmann, ${ }^{b, e}$ T. Perneger ${ }^{a, b}$}

a Institut de médecine sociale et préventive, Université de Genève

b Unité qualité des soins, Hôpitaux Universitaires de Genève

c Unité de développement et de recherche en éducation médicale

d Département de médecine communautaire,

Hôpitaux Universitaires de Genève

e Hôpital de Gériartrie, HUG

f Département de médecine interne, HUG

g Division d'anesthésiologie, HUG

\section{Résumé}

Cet article identifie les raisons de l'intérêt actuel pour la qualité des soins, décrit les modèles conceptuels de la qualité appliqués aux soins médicaux, et identifie les notions de système, de processus, et de boucle d'apprentissage en tant qu'éléments centraux de la gestion efficace de la qualité des soins à l'hôpital.

\section{La qualité des soins: un thème émergent}

Depuis plus d'une décennie, l'intérêt porté à la qualité des soins par les professionnels, les pouvoirs publics, les compagnies d'assurance ou les patients ne cesse de croître. Plusieurs phénomènes contribuent à cet intérêt:

1. La médecine est devenue progressivement plus efficace, mais aussi plus complexe et plus dangereuse, et les récits de mésaventures, erreurs, et autres résultats défavorables se multiplient, aussi bien sur les manchettes des quotidiens («Fausse jambe amputée») que dans des rapports d'experts (par d'exemple, un récent rapport américain intitulé "To err is human" [1] révèle que l'erreur médicale pourrait être responsable aux USA d'un nombre de décès supérieur à celui des accidents de la route).

2. L'augmentation des coûts des soins attire inévitablement l'attention des organismes payeurs (assurances, état, etc.), qui ne veulent pas couvrir des prestations qui seraient inutiles ou mal justifiées. En Suisse, c'est la loi sur l'assurance maladie (LAMal) de 1994 qui a introduit la préoccupation de la qualité des soins dans les textes réglemen-

Correspondance:

Dr Philippe Chastonay

Centre médical universitaire

Institut de médecine sociale et préventive

Rue Michel-Servet

CH-1211 Genève 4 taires, par le biais des conditions de remboursement. Ainsi, l'article 32 stipule que pour être remboursées, les prestations de soins doivent être "efficaces, appropriées et économiques», l'article 39 exige que les hôpitaux disposent d'un personnel qualifié, d'équipements médicaux appropriés, et fournissent les médicaments de façon adéquate, et l'article 58 autorise le Conseil Fédéral à prendre des mesures variées destinées à garantir la qualité des soins.

3. De nombreuses études ont montré une grande variabilité dans les pratiques médicales, que n'expliquent guère les caractéristiques des patients ou des populations servies. Par exemple, le taux de certaines interventions chirurgicales (tonsillectomies, hystérectomies) peut varier du simple au quintuple pour des populations pourtant comparables, et le coût des soins médicaux ambulatoires par assuré est 2,5 fois plus élevé à Genève qu'à Appenzell. Même s'il est difficile de dire qui a raison et qui a tort, force est de constater que tout le monde ne peut pas avoir raison en même temps. Ce constat est notamment à l'origine des évaluations systématiques des connaissances médicales qui visent à aider les cliniciens à prendre des décisions fondées sur des faits plutôt que sur des opinions ("evidence-based medicine»).

4. Plus généralement, la société a changé, la médecine a perdu de son prestige, et une attitude critique du public envers la médecine est désormais considérée comme légitime. Les patients d'aujourd'hui questionnent les décisions de leur médecin, en changent s'ils ne sont pas contents, exigent de la disponibilité et des résultats, forment des associations pour défendre leurs intérêts, bref, se comportent en clients.

\section{Cadre conceptuel}

Il n'existe pas de définition universellement acceptée de la qualité des soins. Pour certains auteurs, notamment ceux qui s'intéressent à la production industrielle, "qualité» est une appréciation globale, équivalente d'«excellence», "conformité aux attentes", "zéro défauts" ou "satisfaction du client".

D'autres estiment que la qualité des soins est multidimensionnelle, et qu'elle implique des notions telles que équité, accessibilité, sécurité, efficacité, efficience, "patient centeredness" (être centré sur le patient), continuité, etc. Une définition fréquemment citée est celle de l'Institut de médecine américain: la qualité des soins dirait «à quel point les services de santé pour les individus et les populations augmentent la probabilité de résultats de santé souhaitables et sont conformes aux connaissances professionnelles actuelles». Des définitions multidimensionnelles sont souvent préférées par professionnels de la santé, mais elles ont le désavantage de tolérer des contradictions. Qualité ne veut pas forcément dire la même chose pour un individu et pour la société, pour le payeur et pour le soignant, etc. 


\section{Figure 1}

Modèle de Donabedian: trois points de vue sur la qualité des soins.

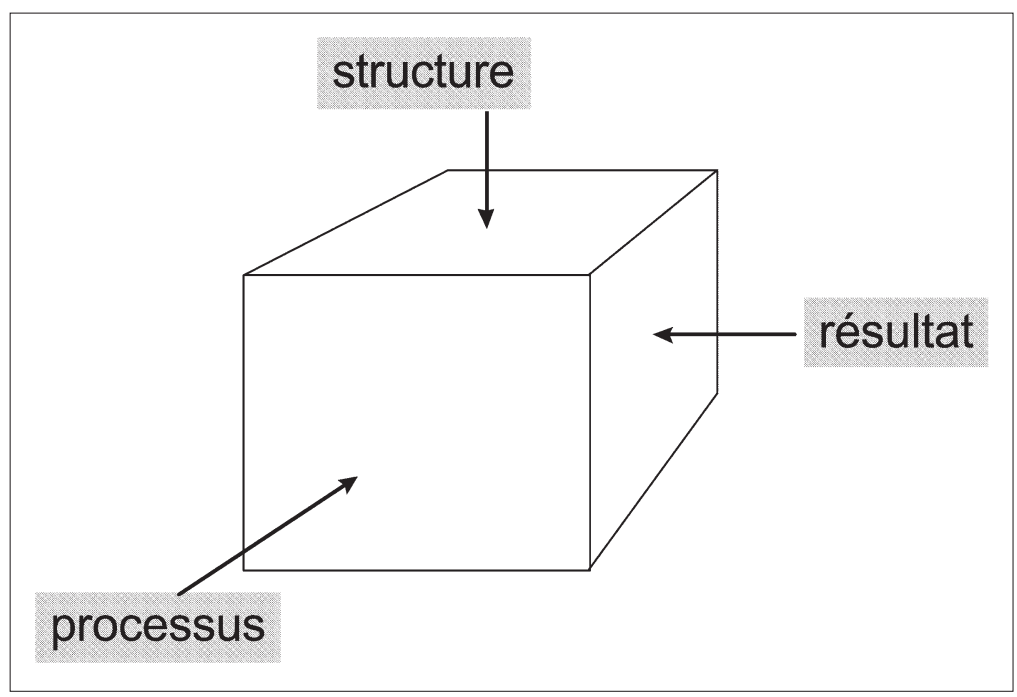

Un autre cadre conceptuel multidimensionnel de la qualité des soins, fréquemment utilisé, a été proposé par Donabedian (fig. 1). Cet auteur distingue trois aspects des soins: les structures, les processus, et les résultats [2].

- les structures correspondent aux ressources mises à disposition pour les soins: dotation en personnel, ses qualifications, équipements, appareils, locaux;

- les processus désignent les soins fournis au patient; on s'intéressera en particulier à leur conformité aux bonnes pratiques professionnelles;

- les résultats des soins comprennent l'état de santé du patient, sa survie, sa qualité de vie, la survenue de complications, etc. Généralement, la satisfaction des patients est également inclue dans cette catégorie.

Cette triade correspond à trois points de vue différents sur le même système complexe des soins: celui $\mathrm{du}$ planificateur, celui du professionnel, et celui du patient. Mais ce cadre ne décrit pas explicitement les articulations entre structures adéquates, bonnes pratiques, et résultats de santé favorables. De ce fait, ce modèle est mieux adapté à l'évaluation de la qualité des soins qu'à son amélioration.

\section{Système ou individu?}

Pendant longtemps, on a estimé que la mauvaise qualité (des soins ou d'autre chose) était due avant tout à l'incompétence ou à la négligence des professionnels. Logiquement, les actions correctives ont consisté à trouver le fautif, l'exhorter à mieux faire, le punir, ou le renvoyer. Or, cette approche est inefficace, particulièrement lorsque l'individu en question ne maîtrise pas l'ensemble des processus concernés. De nos jours, dans les secteurs de haute technologie (navette spatiale, centrale nucléaire, etc.), on estime que les individus sont le plus souvent très compétents et motivés à bien faire, mais qu'ils ne sont pas infaillibles. En effet, la performance des individus dépend du contexte: équipements, locaux, procédures, modes de fonctionnement, flux d'information, style de management, contraintes réglementaires, etc. Dès lors, la maîtrise de la qualité nécessite une réflexion sur le fonctionnement du système dans son ensemble, et doit tenir compte du fait que les individus se trompent occasionnellement [3].

Les soins hospitaliers correspondent bien à ce modèle $[4,5]$. La prise en charge d'un patient admis à l'hôpital débute par le recueil d'informations administratives (identité du patient, assurance maladie, etc.), se poursuit par un premier examen clinique qui évalue la gravité de la situation et la priorité du cas, puis par un examen clinique plus complet qui cerne plus précisément le problème, avec l'aide d'examens complémentaires ou d'avis de spécialistes. Une décision thérapeutique est alors prise; le patient est inscrit au programme opératoire. L'opération, à laquelle contribuent plusieurs spécialistes, est effectuée, suivie d'un passage en salle de réveil puis d'un retour du patient à l'étage, où un traitement de réhabilitation est entrepris. Le patient quitte l'hôpital, après avoir effectué au préalable certaines formalités administratives. De surcroît, cette prise en charge nécessite d'autres activités plus éloignées du patient. Des soignants doivent être formés et disponibles, des équipements acquis et entretenus, des consommables mis à disposition, des logiciels développés, installés sur des postes de travail maintenus en état de marche, des budgets obtenus et alloués. Ainsi la prise en charge d'un patient correspond à la mise en œuvre d'un système complexe dont le fonctionnement dépend de multiples paramètres. En conséquence, les actions correctives doivent porter en priorité sur les facteurs systémiques qui déterminent la qualité de la prise en charge des patients.

\section{Trois exemples}

Trois exemples des Hôpitaux universitaires de Genève illustrent ce propos. On sait que la prise de bêta-bloquants permet de diminuer la mortalité des patients ayant subi un infarctus du myocarde. Toutefois, une enquête réalisée dans les HUG a démontré que seuls $38 \%$ de ces patients quittaient l'hôpital avec une prescription de béta-bloquants. L'intervention corrective n'a pas consisté à punir les récalcitrants, mais à diffuser des recommandations de bonne pratique, développées de concert par les cardiologues et les internistes, et traduites par une fiche d'attitude glissée systématiquement dans les dossiers des patients admis pour infarctus. Cette intervention a permis de doubler la proportion de patients sortant avec une prescription de bêta-bloquants [6].

Le second exemple concerne la prévention des infections acquises à l'hôpital (dites nosocomiales). Ces infections sont fréquemment dues à une transmission 


\section{Tableau 1}

Exemples de processus.

\begin{tabular}{lll}
\hline processus & élément d'entrée & élément de sortie \\
\hline déterminer la glycémie & échantillon de sang & glycémie \\
\hline prescrire un traitement & pathologie & prescription \\
\hline & indications & \\
\hline contre-indications & \\
\hline anesthésier un patient & endoscope souillé & endoscope désinfecté \\
\hline
\end{tabular}

de germes pathogènes par les mains des soignants, et le lavage des mains est la clé de voûte de leur prévention. En 1994, une enquête a montré que le lavage des mains n'avait lieu que dans $48 \%$ des situations où il était nécessaire. L'intervention corrective a comporté d'une part une campagne de sensibilisation par des posters, d'autre part la diffusion de flacons de poche de solution alcoolique désinfectante, qui permet d'éliminer les germes plus rapidement que le lavage à l'eau et au savon. Trois ans plus tard, la conformité du lavage/désinfection des mains avait atteint $68 \%$ [7].

Le troisième exemple concerne la communication avec des patients ambulatoires non-francophones. Soigner un patient avec qui on ne peut communiquer efficacement est frustrant aussi bien pour le médecin que pour le patient, et peut mener à des malentendus, des décisions inadaptées, l'inobservance du traitement par le patient, etc. Une équipe de la Policlinique de médecine a organisé un réseau d'interprètes, rédigé un guide expliquant les principes de communication avec interprètes, et a formé les médecins à ces techniques. Cette intervention a permis d'améliorer de façon significative la satisfaction des patients non-francophones envers la communication avec leur médecin.

\section{Figure 2}

Représentation schématique d'un processus.

méthode:
él. d'entrée:
échantillon fabriquant

\section{Processus}

Dans chacun des exemples ci-dessus, on a amélioré un aspect particulier du fonctionnement de l'hôpital: prescription de médicaments, respect des règles d'hygiène, communication avec les patients. Chacun de ces aspects peut être décrit comme un "processus", à savoir la transformation (au sens large) d'un "élément d'entrée» en un "élément de sortie».

Cette transformation dépend de plusieurs facteurs. Elle se déroule dans un lieu donné, est réalisée par une ou plusieurs personnes, selon une méthode définie, à l'aide d'équipements et de produits consommables, et en tenant compte d'éventuelles contraintes (par exemple éthiques ou légales). Ainsi dans un laboratoire de sérologie transfusionnelle, le technicien qui détermine un groupe sanguin à partir d'un échantillon de sang et à l'aide de divers matériels et réactifs en appliquant une procédure, conduit un processus (fig. 2).

\section{Boucle d'amélioration de la qualité}

La maîtrise d'un système complexe ne peut être obtenue que si l'ensemble des processus qui le composent sont eux-mêmes maîtrisés. Compte tenu du grand nombre de processus impliqués dans les soins et des variations qui peuvent les affecter, il s'agit d'une tâche difficile qui nécessite d'associer en permanence des opérations de prévention et de correction.

Un modèle permet de décrire cette activité: la boucle d'amélioration de la qualité (fig. 3), également connue sous le nom de cycle PDCA (Plan, Do, Check, Act) [8]. Dans ce modèle, la planification a pour but de prévenir le dysfonctionnement d'un processus. Elle implique la réalisation d'un référentiel qui sera utilisé pour guider l'activité lors de l'exécution du processus. Malgré cela, des dysfonctionnements pourront survenir. Leur détection nécessite que le processus fasse l'objet d'une évaluation continue ou périodique. Une analyse de ces dysfonctionnements doit ensuite être conduite afin d'en déterminer les causes et de mettre en œuvre les actions correctives appropriées.

La planification d'un processus doit déboucher sur l'établissement d'un référentiel (ou procédure). Ce document décrit le processus et précise les caractéristiques de ses éléments de sortie, la manière de les obtenir, les contrôles à effectuer, les équipements à employer, les personnes habilitées à le conduire ainsi que les informations à saisir pour rendre compte de son fonctionnement. Il peut comprendre:

- des instructions de travail qui décrivent les différents gestes à accomplir pour obtenir un résultat donné (exemple: pose d'une sonde urinaire);

- des recommandations pour la pratique clinique qui précisent les étapes d'un raisonnement clinique (exemple: évaluation du risque cardiovasculaire per-opératoire); 
Figure 3

La boucle d'amélioration de la qualité.

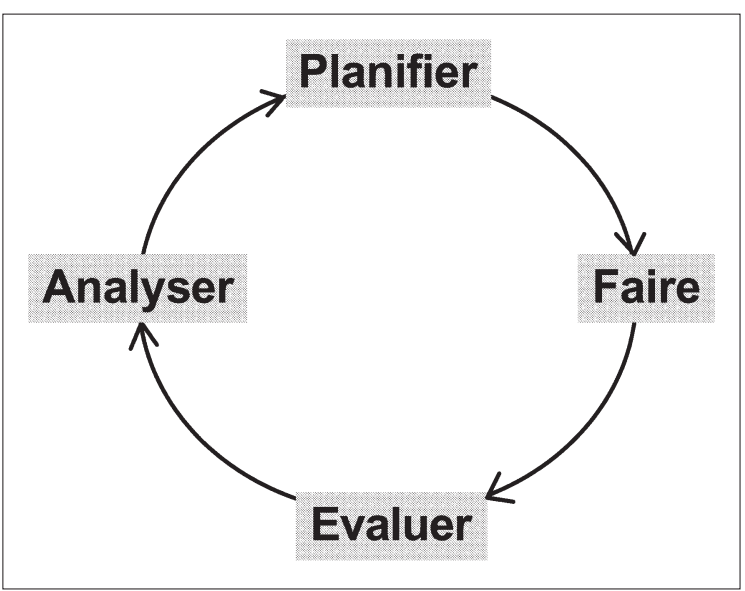

- des diagrammes ou algorithmes qui représentent graphiquement les opérations à réaliser, leur déroulement au cours du temps, les différents nœuds de décision, etc.

En médecine, cependant, la rédaction d'un référentiel peut s'avérer délicate. En effet, les raisonnements conduisant à un diagnostic ou à la sélection d'un traitement sont complexes, à cause d'une connaissance médicale incomplète, de la grande variété des maladies et des traitements, de la nature probabiliste des prédictions (les symptômes n'est pas toujours univoques et des risques d'erreur sont possibles), et de la nécessité de concilier des critères de décision multiples (les traitements envisageables présentent souvent des avantages et des inconvénients, ce qui complique les choix).

Quant à l'évaluation des processus de soins, différents mécanismes de surveillance peuvent être envisagés:

\section{Figure 4}

Evolution du nombre de gazométries par patient-jour aux soins intensifs de chirurgie.

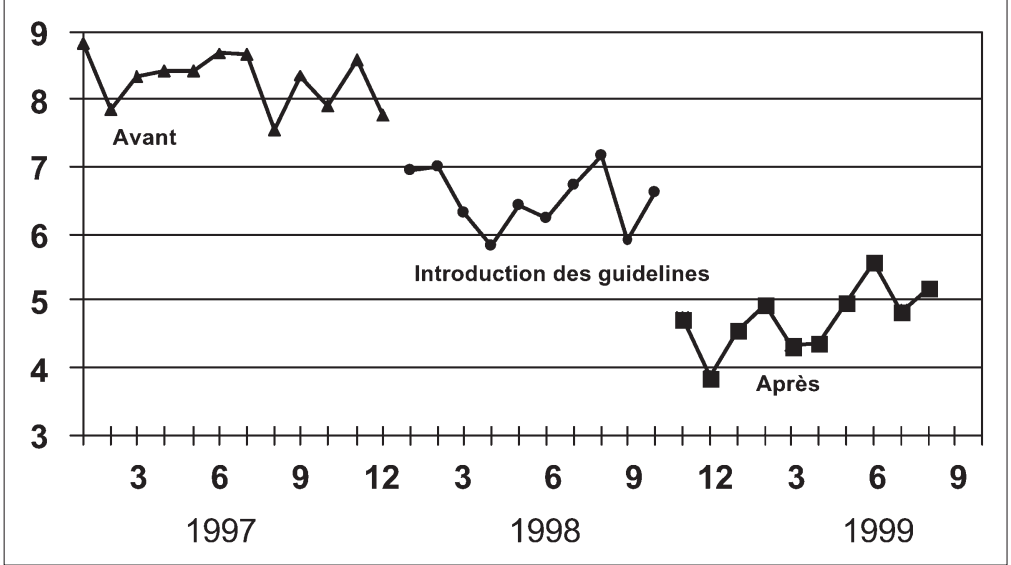

- des contrôles destinés à vérifier l'adéquation des éléments de sortie d'un processus. Par exemple, le positionnement d'une prothèse peut être vérifié systématiquement par une radiographie;

- des audits qui vérifient périodiquement que le processus s'est déroulé conformément au référentiel. Par exemple, on peut contrôler si l'administration de tel médicament dangereux respecte les mesures de sécurité préconisées;

- des recueils d'incidents qui consistent à rapporter toute anomalie ou dysfonctionnement observés au cours des soins. Il peut s'agir par exemple d'erreurs d'administration de médicaments, ou de la perte d'un dossier médical;

- des enquêtes de satisfaction qui permettent de s'assurer périodiquement que ce qu'on fait répond bien aux attentes des clients. Dans un hôpital, on peut évaluer la satisfaction des patients sur différents aspects de la prise en charge.

Lorsqu'un contrôle, audit, relevé d'incident ou enquête de satisfaction révèle un dysfonctionnement, il faut rechercher l'origine du problème et de proposer des actions correctives. Un exposé détaillé des méthodes d'analyse des dysfonctionnements dépasse le cadre d'un exposé introductif. Dans tous les cas, l'identification des causes du dysfonctionnement doit conduire à planifier à nouveau le processus et modifier son référentiel. Ensuite, la boucle d'amélioration repart pour un tour.

\section{Exemple de boucle d'apprentissage: prescription des gazométries aux soins intensifs}

Une enquête préliminaire conduite aux Soins intensifs de chirurgie des HUG a montré qu'environ la moitié des gazométries prescrites pourrait être inutile. Afin de corriger ce problème, une intervention destinée à modifier le comportement de prescription des médecins et des infirmières est engagée. Elle consiste en la diffusion de règles de prescription des gazométries présentées sous la forme d'un algorithme (référentiel) et en l'évaluation périodique, un jour par mois, du nombre moyen de gazométries par patient et par jour (contrôle) et de la conformité des prescriptions aux règles établies (audit). L'évaluation de la situation initiale est conduite rétrospectivement sur une période de 10 mois (phase initiale). L'introduction de la première version de l'algorithme est suivie prospectivement durant 10 mois (phase pilote) puis l'algorithme est réévalué. Une nouvelle version est proposée et fait là encore, l'objet d'un suivi prospectif d'une durée de 10 mois (phase de consolidation). Durant les trois phases, la mortalité ainsi que le nombre moyen d'incidents par patient et par jour sont également mesurés.

Les résultats (fig. 4) montrent qu'entre la phase initiale et la phase pilote, le nombre moyen de gazométries par patient et par jour passe de 8,2 à 6,5 et la conformité au référentiel monte de $53 \%$ à $68 \%$. Entre 
la phase pilote et la phase de consolidation, le nombre moyen de gazométries par patient et par jour passe de 6,5 à 4,8 tandis que la conformité au référentiel monte de $68 \%$ à $80 \%$. Parallèlement la mortalité et le nombre moyen d'incidents par patient et par jour restent inchangés.

\section{Conclusion}

Les soins médicaux sont délivrés par un système complexe mais fragile, qui n'a pas toujours été pensé pour fonctionner de façon optimale. Une réflexion approfondie sur le fonctionnement de ce système, sur la nature de ses défaillances et de leurs causes ainsi que sur les moyens aptes à les prévenir est indispensable. La prise en considération la complexité des systèmes de soins, l'analyse critique des processus qui les constituent, et la mise en œuvre de boucles d'apprentissage (planifier-faire-évaluer-analyser) sont trois composantes d'une approche constructive de la qualité des soins.

\section{Références}

1 Kohn LT, Corrigan J, Donaldson M (eds.). To err is human. Building a safer health system. Washington, DC: National Academy Press; 2000.

2 Donabedian A. The quality of care. How can it be assessed? JAMA 1988;260:1743-8.

3 Nolan TW. System changes to improve patient safety. Br Med J 2000;320:771-3.

4 Berwick D. Continuous improvement as an ideal in health care. N Engl J Med 1989;320:53-6.

5 Leape L. Error in medicine. JAMA 1994;272:1851-7.

6 Sarasin F, Maschiangelo ML, Schaller MD, Heliot C, Mischler S, Gaspoz JM. Successful implementation of guidelines for encouraging the use of beta blockers in patients after myocardial infarction. Am J Med 1999;106:499-505.

7 Pittet D, Hugonnet S, Harbarth S, Mourouga P, Sauvan V, Touveneau S, et al. Effectiveness of a hospital-wide programme to improve compliance with hand hygiene. Lancet 2000;356:1307-12.

8 Deming WE. Out of the crisis. Cambridge MA: MIT Press, 1986. 